\title{
Effects of the noradrenergic agonist clonidine on temporal and spatial attention
}

\author{
Sander Nieuwenhuis • I. Caroline van Nieuwpoort • \\ Dick J. Veltman • Madeleine L. Drent
}

Received: 18 October 2006 / Accepted: 5 March 2007 /Published online: 13 April 2007

(C) Springer-Verlag 2007

\begin{abstract}
Rationale Recent theories posit an important role for the noradrenergic system in attentional selection in the temporal domain. In contrast, the spatially diffuse topographical projections of the noradrenergic system are inconsistent with a direct role in spatial selection.

Objectives To test the hypotheses that pharmacological attenuation of central noradrenergic activity should (1) impair performance on the attentional blink task, a task requiring the selection of targets in a rapid serial visual stream of stimuli; and (2) leave intact the efficiency of the search for a target in a two-dimensional visuospatial stimulus array.

Materials and methods Thirty-two healthy adult human subjects performed an attentional blink task and a visual search task in a double-blind, placebo-controlled, betweensubject study investigating the effects of the $\alpha 2$ adrenoceptor agonist clonidine $(150 \mu \mathrm{g}$, oral dose).
\end{abstract}

\footnotetext{
S. Nieuwenhuis $(\square)$

Department of Psychology, Leiden University,

Wassenaarseweg 52,

2333 AK Leiden, The Netherlands

e-mail: snieuwenhuis@fsw.leidenuniv.nl

S. Nieuwenhuis

Department of Psychology, Vrije Universiteit,

Van der Boechorststraat 1,

1081 BT, Amsterdam, The Netherlands

I. C. van Nieuwpoort $\cdot$ M. L. Drent

Department of Endocrinology, VU University Medical Center,

P.O. Box 7057, 1007 MB, Amsterdam, The Netherlands

D. J. Veltman

PET Center, VU University Medical Center,

P.O. Box 7057, 1007 MB, Amsterdam, The Netherlands
}

Results No differential effects of clonidine vs placebo were found on the attentional blink performance. Clonidine slowed overall reaction times in the visual search task but did not impair the efficiency of the visual search.

Conclusions The attentional blink results are inconsistent with recent theories about the role of the noradrenergic system in temporal filtering and in mediating the attentional blink. This discrepancy between theory and data is discussed in detail. The visual search results, in combination with previous findings, suggest that the noradrenergic system is not directly involved in spatial attention processes but instead can modulate these processes in an indirect fashion.

Keywords Norepinephrine - Clonidine $\cdot$ Locus coeruleus . Attention $\cdot$ Attentional blink $\cdot$ Visual search

\section{Introduction}

The locus coeruleus-norepinephrine (LC-NE) system is one of several brainstem neuromodulatory nuclei with widely distributed, ascending projections to the neocortex (Berridge and Waterhouse 2003). The conventional view of $\mathrm{NE}$ is that it has broad, nonspecific functions such as regulating arousal (Jouvet 1969). However, recent studies suggest that NE may have more specific cognitive functions, which are nevertheless closely related to the concept of arousal (Robbins 1997; Aston-Jones et al. 2000; Yu and Dayan 2005; Chamberlain et al. 2006). In particular, it has been proposed that NE has a key role in facilitating the responses to decision-making processes and in regulating the balance between exploitation and exploration (Aston-Jones and Cohen 2005). 
Recent views on the role of the LC-NE system in decision making have been based on neuronal recordings of the primate LC during the performance of simple decisionmaking tasks. These recordings have indicated that under alert conditions (i.e., conditions typical of human subjects in our experiments), LC phasic responses are selectively observed after task-relevant and otherwise motivationally significant (e.g., highly salient or infrequent) stimuli (Aston-Jones et al. 2000). Furthermore, stimulus-elicited phasic LC bursts reliably precede (by $200 \mathrm{~ms}$ ) and are closely coupled in time with the behavioral response (Bouret and Sara 2004; Clayton et al. 2004). The noradrenergic innervation associated with such LC responses results in a system-wide, transient increase in the responsivity of efferent target neurons, which is thought to facilitate processing in response to the eliciting stimulus (Berridge and Waterhouse 2003). These observations have led to the suggestion that the LC phasic response can be thought of as a temporal attentional filter (1) that selects for the occurrence (i.e., timing) of task-relevant (Aston-Jones and Cohen 2005) or unexpected (Dayan and Yu 2006) events, much like cortical attentional systems filter the content (e.g., spatial characteristics or color) of stimuli and (2) that facilitates the processing of and responding to these stimuli to help optimize task performance (Aston-Jones and Cohen 2005; Nieuwenhuis et al. 2005a; see also Coull 1994).

In the current study, we evaluated the following two hypotheses: (1) According to the temporal filtering hypothesis outlined above, the LC-NE system should be involved in temporal attention modulations operating at the phasic time scale (e.g., in the order of tens of seconds); (2) In contrast, because of the spatially nonspecific pattern of LC projections to the forebrain, the LC-NE system cannot be directly involved in spatial attention shifts. That is, although the LC-NE system projects to multiple brain areas, including many known to be involved in spatial attention, the diffuse character of these projections is inconsistent with the direct biasing of attention in any specific direction (i.e., the LC does not "know" the relevant spatial location). To test these two hypotheses, we contrasted, in human subjects, the effects of a noradrenergic drug on measures of temporal and spatial attention. Most previous studies examining the functional significance of the LC-NE system have employed drugs acting on $\alpha 2$ receptors. In this study, we used the $\alpha 2$ adrenoceptor agonist clonidine, which at low doses decreases LC firing and attenuates the release of NE from axon terminals (Svensson et al. 1975).

Previous studies have examined the effects of clonidine on temporal and spatial attention in a visuospatial cueing task. In this task, subjects are required to respond as quickly as possible to a series of stimuli presented to the left or right of fixation. Each stimulus is preceded by a cue indicating at which location the imperative stimulus is most likely to appear, and subjects are instructed to make use of this information by switching their attention to the location indicated by the cue. The comparison of reaction times on validly versus invalidly cued trials (the "validity effect") provides an index of the efficiency of cue-induced spatial attention shifts. Fernandez-Duque and Posner (1997) extended this paradigm by including and contrasting reaction times on neutral-cue trials and no-cue trials (the "alerting effect"), which provides an index of the transient increase in responsivity brought about by the mere presentation of the cue. The alerting effect thus serves as a measure of temporal (cue-evoked) changes in attention to the imperative stimulus.

Clonidine has been found to reduce the size of the alerting effect in humans (Coull et al. 2001) and monkeys (Witte and Marrocco 1997), suggesting that the temporal attentional effects of cues are mediated in part by the noradrenergic system. In contrast, studies have reported mixed results regarding the influence of clonidine on the validity effect: Two studies have reported a reduced validity effect (Clark et al. 1989; Coull et al. 2001), and one study has reported no effect of clonidine (Witte and Marrocco 1997). However, it is hard to interpret clonidine-related reductions in the validity effect, because they may reflect either a direct involvement of the noradrenergic system in spatial attention processes or an interaction between the alerting and attention-directing (i.e., spatial) effects of cues. Specifically, by increasing the responsivity of cortical representations after cue presentation, LC-induced phasic NE release can enhance the effects of target selection by location (e.g., the top-down modulation by prefrontal cortex), resulting in an increased validity effect. The effects of this amplification mechanism would be diminished by clonidine.

In contrast to previous studies, we used separate tasks for measuring the effects of clonidine on temporal and spatial attention: an attentional blink task, which requires the selection of targets in time and has no spatial component, and a visual search task, which requires the selection of targets in space but without the help of alerting cues.

In the attentional blink task, each trial starts with a rapid serial visual presentation (RSVP) stream containing two target stimuli (T1 and T2) and multiple distractors, each presented for about $100 \mathrm{~ms}$. At the end of the RSVP stream, subjects are required to report the identity of $\mathrm{T} 1$ and $\mathrm{T} 2$.

The critical finding in this task is that subjects are heavily impaired at the identification of $\mathrm{T} 2$ for a few hundred milliseconds after correct identification of T1. This deficit, known as the "attentional blink," is usually most severe around 200-400 ms (or two to four items) after T1, after which, performance gradually recovers (Raymond 
et al. 1992; Chun and Potter 1995). In contrast, if T2 follows T1 without intervening distractors (at "lag 1"), performance on $\mathrm{T} 2$ is often (partially) spared (Raymond et al. 1992; Hommel and Akyürek 2005); this phenomenon has been labeled "lag-1 sparing." These and other findings suggest that the attentional blink task reveals important clues about the time course of fine-scale fluctuations in attention (Olivers 2007).

Recently, a theory has been proposed that explains the attentional blink in terms of the temporal dynamics of the LC-NE system (Nieuwenhuis et al. 2005b). The theory was inspired by the observation that the timing of the attentional blink coincides with the refractory period in LC activity that follows an LC phasic response to target stimuli. Specifically, although NE potentiates processing in cortical areas, local NE release within the LC is autoinhibitory because of the noradrenergic action at presynaptic and dendritic $\alpha 2$ autoreceptors (Aghajanian et al. 1977). This autoinhibition results in a period after the LC phasic response ( 200-450 ms after the eliciting stimulus), during which subsequent LC phasic discharge is rarely observed. According to the theory, the attentional blink may be mediated by this momentary unavailability of the LC phasic response (and attendant noradrenergic potentiation of information processing) after the $\mathrm{LC}$ response to $\mathrm{T} 1$. In other words, if $\mathrm{T} 2$ is presented during the LC refractory period associated with $\mathrm{T} 1$, it will not receive the benefit of NE-mediated facilitation and is therefore more likely to remain unidentified. A computational model implementing this theory was shown to accurately simulate the time course of the attentional blink, including lag-1 sparing (Nieuwenhuis et al. 2005b). In addition, the theory offers an account of the close relationship of the attentional blink to the P3 component of the event-related potential, an electrophysiological correlate of the phasic NE release (Nieuwenhuis et al. 2005a).

To assess the effect of clonidine on spatial attention, we used a visual search task. On each trial, subjects searched for a target among multiple distractors in a visual search array and indicated as quickly as possible whether the target was present or absent. The number of distractors (i.e., set size) was systematically varied across trials. In one condition (single-feature search), the target was defined by a salient basic feature, such that search was efficient and the number of distractors had little influence on reaction times. In another condition (conjunction search), the target was defined by a conjunction of two features, such that search was inefficient, and reaction times increased linearly with the number of distractors - as if each item needed to be examined in turn. In this task condition, the efficiency of spatial attention processes is expressed in the function relating search reaction times to set size: The more visual attention is impaired, the steeper the slope of this function (as expressed in an increased effect of set size; Tales et al. 2002).

Our predictions with regard to the effects of clonidine were the following: First, we expected that clonidine would impair performance on the attentional blink task, in particular through its stimulation of inhibitory $\alpha 2$ auto receptors in the LC. In accordance with the temporal filtering hypothesis, we expected that the clonidine-induced reduction in noradrenergic activity would lead to an impaired identification of $\mathrm{T} 1$ but especially of $\mathrm{T} 2$, because it should presumably exacerbate the lack of NE associated with T2 processing under normal conditions (Nieuwenhuis et al. 2005b). Second, aside from the well-documented main effect of clonidine on response speed (i.e., general slowing; e.g., Tiplady et al. 2005), we expected no drug effects on visual search efficiency, as indexed by the effects of distractor set size. This latter prediction was based on the notion that the effects of NE are topographically diffused and hence should not affect the efficiency of selection in the spatial domain.

\section{Materials and methods}

\section{Subjects}

Thirty-two healthy young adults, aged 18-25 years, took part in a single experimental session in return for 60 . Only subjects with a systolic blood pressure above $100 \mathrm{mmHg}$ and a diastolic blood pressure above $70 \mathrm{mmHg}$ were included in the study. All subjects underwent a medical screening and were considered to be in satisfactory health. The use of medication that could interfere with clonidine was stopped the day before. All subjects with color blindness were excluded from the study.

Subjects received an oral dose of $150 \mu \mathrm{g}$ of clonidine or placebo in a double-blind, between-subjects design. Clonidine has well-established antihypertensive properties; therefore, blood pressure and heart rate were monitored for subject safety. Measurements were taken every $15 \mathrm{~min}$ starting from $t=-15$ until $t=120$ and every $30 \mathrm{~min}$ from $t=120$ until $t=240$.

The clonidine group ( $N=16$, eight women, $M$ age 21.3) and the control group ( $N=16$, eight women, $M$ age 21.3) had similar mean ages. We also verified that the groups were similar in terms of intellectual functioning as assessed with the three-subtest version of the Groninger Intelligence Test (GIT), a reliable indicator of the full-scale IQ (Luteijn 1966). Estimated IQ scores were 117.1 for the clonidine group and 116.8 for the control group. The visual-search task data from one subject from the clonidine group were discarded because of an accidental failure to comply with 
the task instructions. The study was approved by the medical ethics committee of the VU University Medical Center and was conducted according to the Declaration of Helsinki. Informed consent was obtained from all subjects before their inclusion in the study.

\section{Procedure}

Each subject was tested at approximately the same time of the day (afternoon). Subjects were instructed to abstain from caffeine, nicotine, and other psycho-active substances from $15 \mathrm{~h}$ before the start of the session and to abstain from alcohol from $15 \mathrm{~h}$ before the start of the session until the day after the session. After the medical screening, subjects received a lactose-filled capsule with either clonidine or placebo. Immediately after that, the subtests of the GIT were administered. The two attentional tasks, described in detail below, were performed between $t=60$ and $t=120 \mathrm{~min}$ posttreatment. Half of the subjects in each group started with the attentional blink task. The other half started with the visual search task. After completion of the tasks, the subjects were debriefed and paid. At $t=240$, subjects were reevaluated and returned home by taxi if blood pressure was (near) normal.

\section{Attentional blink task}

Stimulus generation and response recording in both tasks were controlled by E-Prime software (Psychology Software Tools, Pittsburgh, PA). Stimuli were presented in black against a light grey background. Each trial started with a fixation cross measuring $0.5 \times 0.5^{\circ}$, presented for $1,000 \mathrm{~ms}$ in the center of the display. Subsequently, the fixation cross was replaced by an RSVP stream of 15-22 uppercase letters, each measuring approximately $0.9 \times 0.9^{\circ}$. Each letter was randomly drawn (without replacement) from the alphabet and presented for $50 \mathrm{~ms}$, followed by a $30-\mathrm{ms}$ blank interval. This relatively rapid pace of stimulus presentation (i.e., $80 \mathrm{~ms}$ between two consecutive stimulus onsets) was chosen to avoid ceiling levels of performance and consequently to increase the sensitivity of the task to individual and group differences. "I," "O," "Q," and "S" were left out as they resemble digits too much. On each trial, two of the letters were replaced with digits, randomly drawn without replacement from the set 2 to 9 . T2 was presented three to six temporal positions from the end of the stream. The temporal distance between $\mathrm{T} 1$ and $\mathrm{T} 2$ was systematically varied from one to five items, corresponding to lags of $80,160,240,320$, and $400 \mathrm{~ms}$. The subject's task was to identify both $\mathrm{T} 1$ and $\mathrm{T} 2$ by typing the digits in order on a standard keyboard after the end of the RSVP stream. Subjects were instructed to guess whenever they failed to identify a digit. The two keyboard entries were followed by the presentation of a feedback stimulus for $150 \mathrm{~ms}$ (e.g., ',+- ' to indicate that $\mathrm{T} 1$ was correct and $\mathrm{T} 2$ was incorrect). After a 1,000-ms blank screen, the next trial started. Each subject started with 15 practice trials, three with each condition, randomly intermixed. This was followed by four blocks of 50 trials each with each block containing ten repetitions of each lag. All task instructions were automated and presented on screen.

\section{Visual search task}

On each trial, subjects searched for a target among multiple distractors in a visual search array. On half of the trials, the target, a vertical red bar, was present in the array. On the other half of the trials, the target was absent. In one condition (single-feature search), the distractors in the search display were vertical green bars of equal size as the target. Because in this case the target is defined by a unique feature (color), it "pops out" from the display, resulting in a fast and efficient search process. In another condition (conjunction search), the distractors were vertical green bars and horizontal red bars. In this case, the target does not consist of a single identifying feature but is defined by a specific conjunction of features (color and orientation), resulting in a more time-consuming and errorprone search process.

Each trial started with a white fixation cross measuring $0.9 \times 0.9^{\circ}$ against a dark background, presented for $500 \mathrm{~ms}$ in the center of the display. Subsequently, the fixation cross was replaced by the search display, which consisted of four, eight, or 16 items that were randomly plotted in the cells of an imaginary $6 \times 6$ matrix $\left(8.7^{\circ}\right.$ horizontally $\times$ $9.6^{\circ}$ vertically) with some random jitter within the cells. The subject's task was to report whether or not the target $\left(0.7 \times 1.3^{\circ}\right)$ was present by giving a response with their left or right index finger using the ' $\mathrm{z}$ ' and ' $\mathrm{m}$ ' keys on the computer keyboard. The keyboard entry was immediately followed a 1,000-ms blank screen after which the next trial started.

Subjects performed four blocks of 96 trials each, with each block containing 16 repetitions of the factorial combination of set size $(4,8$, or 16$)$ and trial type (target present or absent) presented in random order. Search condition (single-feature search or conjunction search) was varied across blocks in an ABBA-order. Half of the subjects started with single-feature search, and the other half started with conjunction search. Subjects received written instructions and 12 practice trials before entering the experimental phase. The task instructions encouraged subjects to respond as quickly as possible while minimizing the number of errors. Performance feedback was provided at the end of each block. All task instructions were automated and presented on screen. 


\section{Results}

Physiological measures

Figure 1 presents for both groups the absolute values of heart rate, systolic, and diastolic blood pressure as observed throughout the experimental session. All three measures showed a significant effect of time (all $P<0.001$ ). Systolic $[F(1,30)=11.8, P=0.002]$ and diastolic blood pressure $[F(1,30)=6.3, P=0.018]$ both showed significant main effects of group. The main effects of time and group were qualified by significant time $\mathrm{X}$ group interactions for systolic $[F(13,390)=9.8, P<0.001]$ and diastolic blood pressure $[F(13,390)=6.1, P<0.001]$.

\section{Attentional blink task}

Figure 2 shows the average T1 accuracy (left panel) and T2 accuracy (right panel; contingent on correct T1 identification) as a function of lag and group. A similar pattern of results was found if T2 accuracy was averaged across correct and incorrect T1 trials. Trials on which T1 and T2 were accurately identified but in the wrong order were treated as correct. As an analysis of variance (ANOVA) showed no significant effects of the order in which the two tasks were performed, this factor was omitted from the reported analyses.

T1 and T2 accuracy were entered in separate two-way mixed ANOVAs with lag and time-on-task (blocks 1,2 vs 3,4 ) as within-subject factors and group as a betweensubject factor. The variable time-on-task was included to
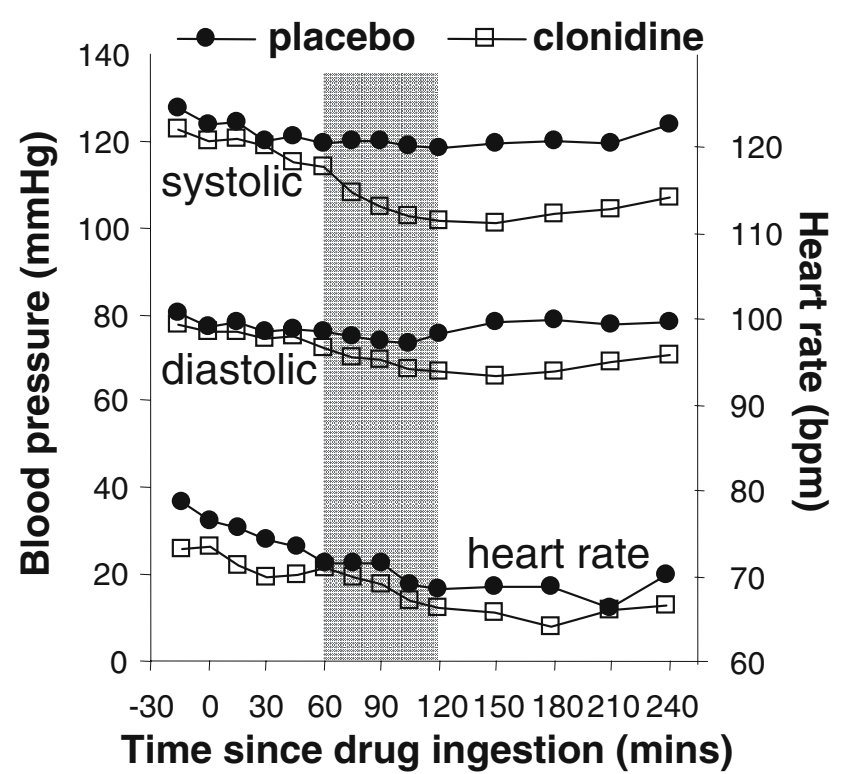

Fig. 1 Systolic and diasystolic blood pressure and heart rate following administration of placebo or $150 \mu \mathrm{g}$ of clonidine (time $=0$ ). The grey shaded area marks the time period during which the two cognitive tasks were administered assess the possibility that a group effect emerged over time. $\mathrm{T} 1$ accuracy showed an increasing trend with lag $[F(4,120)=$ 12.3, $P<0.001]$. The clonidine group $(79.5 \%$ correct $)$ performed slightly worse on $\mathrm{T} 1$ than the placebo group $(84.6 \%)$, but the main effect of group did not reach significance $[F(1,30)=2.0, P=0.17]$. The lag $\mathrm{X}$ group interaction was not significant $[F(4,120)=1.0, P=0.39]$.

The two T2 accuracy curves show a pattern that is characteristic of attentional blink research: lag-1 sparing, followed by a drop in performance for lags 2, 3, and 4 (i.e., the attentional blink), and followed by the initial recovery of performance at lag 5 , at least in the clonidine group. This pattern was expressed in a significant effect of lag $[F(4$, $120)=43.2, P<0.001]$. The most important for the present purposes is the finding that $\mathrm{T} 2$ accuracy did not reliably differ between the two groups $[F(1,30)<1]$. Furthermore, although at lag 4 there was a sizeable numerical effect of group in the expected direction, the lag $\mathrm{X}$ group interaction was not significant $[F(4,120)=1.2, P=0.30]$. Time-on-task did not reliably affect $\mathrm{T} 1$ or $\mathrm{T} 2$ performance or interact with other variables.

In a separate analysis, we calculated the average T2 performance across lags $3-5$ as a summary measure of attentional-blink magnitude and examined group differences in this measure. The two groups did not differ in attentional-blink magnitude, and performance at lags 3-5 did not improve with time-on-task [both $F(1,30)<1$ ]. Interestingly, there was a reliable interaction between group and time-on-task $[F(1,30)=4.9, P=0.034]$ : Whereas performance of the placebo group deteriorated over time (66.6 vs $62.2 \%$ correct; $P=0.65)$, performance of the clonidine group improved slightly (60.3 vs $62.6 \% ; P=0.28$ ).

In a final analysis, we examined the percentage of order reversals at lag 1 , the phenomenon that the two targets, when immediately succeeding each other, are often identified correctly but reported in the wrong order (Hommel and Akyürek 2005). The percentage of such order reversals was roughly the same in the placebo group (34.4\%) and in the clonidine group $[35.4 \% ; t(30)<1]$.

Visual search task: single-feature search

The total number of items in the search display (set size) was varied from trial to trial, allowing us to derive the function relating reaction time (RT) to set size. The slope of this function measures the cost for adding additional items to the display and is often interpreted as "search efficiency," with steeper slopes indicating slower, less efficient search. As expected and illustrated in Fig. 3, slopes in the singlefeature search condition were close to zero [average $1.6 \mathrm{~ms} /$ item; $t(30)=2.6, P=0.014]$.

Correct RTs were entered in a three-way mixed ANOVA with target presence (target present vs target absent) and set 
Fig. 2 Average identification accuracy for the first $(T 1$; left panel) and second (T2; right panel) of the two targets in the attentional blink task as a function of group (clonidine vs placebo) and the lag between $\mathrm{T} 1$ and $\mathrm{T} 2$. As is usual, T2 accuracy is reported contingent on accurate identification of $\mathrm{T} 1$

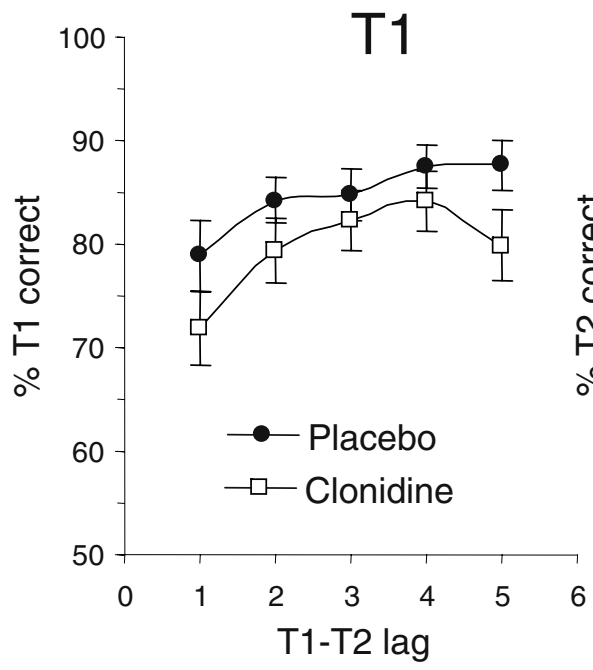

size $(4,8$, or 16$)$ as within-subject factors and group as between-subject factor. Despite the relatively shallow RT slopes, the main effect of set size was significant $[F(2,58)=$ $6.3, P=0.006]$. Furthermore, the clonidine group was overall slower than the placebo group $[F(1,29)=5.6, P=0.025]$. Group did not interact with target presence $[F(1,29)=1.2$, $P=0.29]$ or set size $[F(2,58)=2.5, P=0.10]$. The three-way interaction was also not significant $[F(2,58)<1]$. Error rates were generally low (average 2.3\%). An ANOVA yielded only a significant main effect of target presence $[F(1,29)=$ $8.9, P=0.006]$, with errors occurring more often on targetpresent trials $(2.9 \%)$ than on target-absent trials $(1.8 \%)$.

Visual search task: conjunction search

As expected, slopes in the conjunction-search condition were sizeable [average $25.0 \mathrm{~ms} /$ item; $t(30)=13.8, P<0.001$ ], indicating that visual search was time consuming and inefficient. A three-way mixed ANOVA yielded significant main effects of target presence $[F(1,29)=31.5, P<0.001]$ and set size $[F(2,58)=152.9, P<0.001]$ and a significant interaction of these two variables $[F(2,58)=26.7, P<0.001]$, indicating that set-size effects were larger for target-absent trials. Importantly, the main effect of group was not significant $[F(1,29)=2.1, P=0.16]$, and group did not reliably interact with any of the task variables (all $F<1$ ). Error rates were again low (average 3.3\%). An ANOVA yielded the same pattern of effects as the RT analysis, indicating that there was no speed-accuracy trade-off, and that there were no significant group differences.

\section{Discussion}

The main findings of the current study can be summarized as follows. First, in contrast to our predictions, clonidine did not have a deleterious effect on attentional blink performance. Although the numerical group differences in $\mathrm{T} 1$ and $\mathrm{T} 2$ accuracy were in the expected direction, these differences were small compared to the sizeable performance differences between the individuals within each group (for a discussion of these individual differences, see Martens et al. 2006). The results from the visual search task were as expected: Although clonidine slowed the overall response speed, it did not affect the efficiency of the visual search for a target in a two-dimensional array of stimuli. Below, we will discuss the implications of these principal findings.
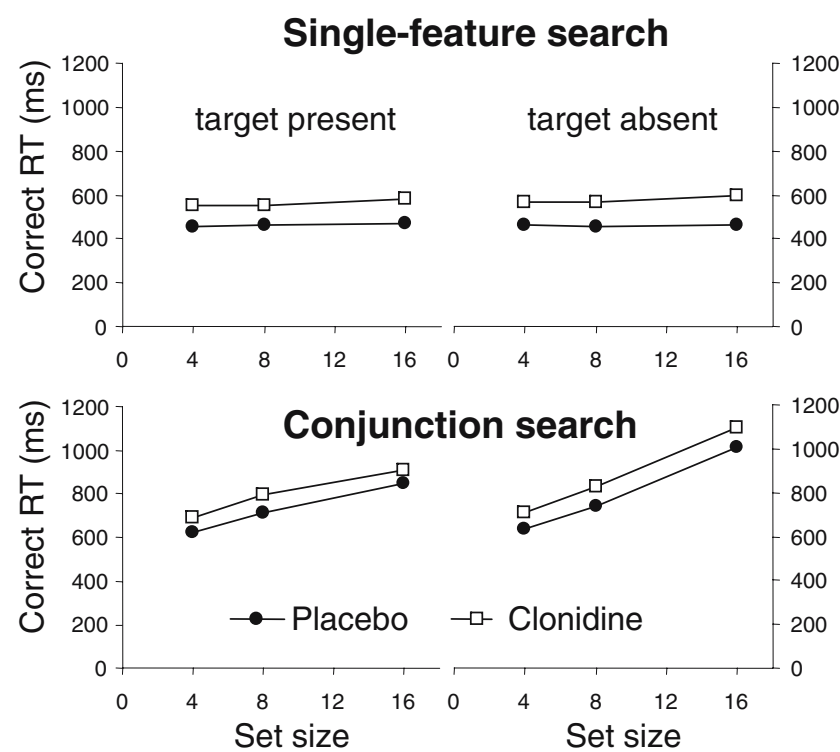

Fig. 3 Average correct reaction times for the clonidine and placebo groups in the visual search task. Top panels show data from the singlefeature search condition, and bottom panels show data from the conjunction search condition. Data for target-present trials are plotted in the left-hand panels, data for target-absent trials are plotted in the right-hand panels 
The absence of a significant drug effect on attentional blink performance appears to be at odds with the temporal filtering hypothesis, which suggests that phasic activity of the LC-NE system is important for the selection of important or infrequent stimuli in the temporal domain (Aston-Jones and Cohen 2005; Dayan and Yu 2006). The attentional blink results also seem inconsistent with the predictions of a recent theory that explains the attentional blink in terms of the temporal dynamics of the LC-NE system (Nieuwenhuis et al. 2005b). One possible explanation for these discrepancies is that the predicted group differences do in fact exist, but that our task and experimental design were not sufficiently sensitive to reveal them. Some critical factors in this regard may be the choice of a between-subject design and the sample size of 16 subjects per group. However, previous studies using between-subject designs, similar group sizes, the same subject population, and the same task have had no difficulty detecting group-levels effect on attentional blink performance of various manipulations aimed at distracting subjects from the RSVP stream (Olivers and Nieuwenhuis 2005, 2006). Indeed, our choice of design was based on these previous studies and on the assumption that the effect of clonidine would be of at least the same size as the effect of these behavioral manipulations. In this context, it is worth noting that the interindividual variance in attentional blink performance was comparable to that in the Olivers and Nieuwenhuis studies, discounting increased variance as a reason for our failure to detect group differences.

A more principled reason for the use of a between-subject design was that subjects can learn to adopt a 'mental state' that greatly benefits attentional blink performance (Olivers and Nieuwenhuis 2006), and we wanted to avoid the transfer of such learning between drug conditions to exclude a potential contaminating source of variance. In addition, Coull et al. (1995a,b) have found that in within-subject designs, the order of treatments (i.e., clonidine before or after placebo) can show complicated interactions with task performance, another source of variance that we aimed to exclude.

Another design feature that may have led to a failure to detect an existing effect is the timing of test administration relative to the treatment ( $t=60-120 \mathrm{~min})$. In particular, the systolic and diastolic blood-pressure time series reveal a maximal drug effect after the test period, suggesting that cognitive effects may have peaked later too. However, there are at least three arguments against this possibility. First, Tiplady et al. (2005) have investigated the time course of effects of $150 \mu \mathrm{g}$ of orally administered clonidine on cognitive test variables and found that such effects were already present at $t=45 \mathrm{~min}$ but were decreased at $t=$ 135 min. Second, our task-block comparisons show that, if anything, the group difference in attentional blink perfor- mance became smaller over time, a trend opposite from that expected if the effects of clonidine needed more time to develop. A third and related argument is that we found no significant interaction between group and task order, corroborating the notion that group effects did not become larger over time. Alternatively, we cannot rule out the possibility that group effects peaked earlier than our test period. However, our choice of test period seems justified by the finding of significant cognitive effects of clonidine in various previous studies using similar test periods (Halliday et al. 1989, 1994; Coull et al. 2001). Thus, our methods and results do not seem to contain any clear indication that the observed null effects reflect a lack of sensitivity. However, it is of course possible that a higher drug dose would yield more robust group differences. This is a possibility that can be addressed in future research (note that such research should take into account that the primary mode of action of clonidine changes with increasing dose; (Arnsten and Cai 1993). At low doses, presynaptic receptors and LC receptors are stimulated, leading to a reduced NE activity. In contrast, at higher doses, postsynaptic receptors are activated, resulting in a boost of NE activity. This has important implications for the expected effects of clonidine on cognitive function).

Another possible explanation for the absence of significant drug effects on attentional blink performance is that the attentional blink may not be mediated by the LC-NE system. This possibility would be particularly bothersome for the theory of Nieuwenhuis et al. (2005b), which directly relates the attentional blink to a refractory period in activity of the LC-NE system. Although there is substantial indirect evidence in support of this theory (e.g., the relationship between the attentional blink and the P3), the basic tenets of the theory are based on cell recordings in animals and are hard to validate directly in humans. Indeed, the current study is perhaps the most direct test to date of a relationship between the attentional blink and the LC-NE system. In this context, it is important to note that another research group has reported preliminary evidence consistent with such a relationship (De Martino et al. 2005). This study, using a between-subject design, found that attentional blink performance was significantly impaired after intake of the B-adrenergic receptor antagonist propranolol (40-mg oral dose compared to placebo). Although this finding is not predicted by the theory of Nieuwenhuis et al. (2005a, b), which relates the attentional blink to the dynamics of inhibitory $\alpha 2$ autoreceptors in the LC, it will be informative to determine to what extent the current findings generalize to other task designs and other noradrenergic drugs. A further avenue for future research will be to test and evaluate the effects of noradrenergic drugs on temporal attention using alternative experimental paradigms, such as the temporal analogue of the spatial cueing paradigm 
(Posner et al. 1980; Coull and Nobre 1998; see Coull et al. 2001). Eventually, accumulating evidence obtained in such human psychopharmacological studies can be used to accommodate theories about the role of NE in temporal attention.

The results from the visual search task were consistent with our predictions: Although clonidine led to a general slowing of response speed, we found no evidence for drug effects on visual search efficiency as indexed by the effects of the distractor set size. This pattern of results was expected based on the notion that the effects of $\mathrm{NE}$ are topographically diffuse (i.e., not selective with regard to any specific spatial representations) and hence should not affect the efficiency of selection in the spatial domain. Previous studies using visuospatial cueing tasks have found that clonidine reduces the cue validity effect (Clark et al. 1989; Coull et al. 2001), which has led to the suggestion that the LC-NE system is directly involved in visuospatial orienting. For example, it has been proposed that the LCNE system plays an important role in the disengagement of visuospatial attention from invalidly cued spaces (Clark et al. 1989; Posner and Petersen 1990). This proposal is consistent with the little available evidence, which suggests that the reduced validity effect after clonidine administration is mainly due to attenuated performance costs on invalidly cued trials. However, we propose an alternative interpretation for these results to reconcile them with our theoretical framework. According to our interpretation, the presentation of the cue causes a spatially nonspecific, transient LC-NE response that boosts the processing of any stimuli presented for a brief period thereafter (i.e., the alerting effect). Although not supported by the current attentional blink findings, this assumption is based on various sources of evidence in the animal literature (Aston-Jones and Cohen 2005; Witte and Marrocco 1997). The cue-induced LC-NE response speeds up cortical spatial selection processes involved in localizing the cue and/or subsequent imperative stimulus. This improves performance on validly cued trials and impairs performance on invalidly cued trials thus increasing the validity effect. Administration of clonidine counteracts this amplifying effect of phasic LC-NE activity thereby reducing the validity effect (note that this hypothesis predicts both improved performance on invalid trials and impaired performance on valid trials). This type of interaction between the alerting effect and spatial selection processes is absent in the visual search task, which has no clear alerting component. Therefore, the current results are consistent with the notion that the LC-NE system is not directly involved in visuospatial orienting but can only indirectly modulate the effects of spatial attention mechanisms implemented elsewhere in the brain.
One drawback of the current study is that we did not include a task that has already been shown to be sensitive to clonidine in previous studies. Therefore, a possible interpretation of the reported null findings is that the administered dose of clonidine failed to affect general cognitive function in our subjects, and that the main effect of drug on visual search RT reflects drug-induced motor slowing rather than a central cognitive deficit. Although we deem this possibility unlikely, given that previous studies using similar doses of clonidine have found significant effects on cognitive function (Coull et al. 2001; Tiplady et al. 2005), a more extensive study with a larger task battery is needed to adequately address this issue.

There is rapidly growing interest in the specific role of the LC-NE system in human cognition (Cohen and AstonJones 2005). Psychopharmacological data from humans will be of critical importance in testing and further developing the increasingly sophisticated hypotheses that are based on neurophysiological observations in animals (e.g., Yu and Dayan 2005). The current research provides a valuable contribution toward a better understanding of the role of the LC-NE system in human attention, both in the spatial and the temporal domain.

Acknowledgment This research was supported by The Netherlands Organization for Scientific Research. We thank Sabrina La Fors for her technical assistance. The reported experiment complies with the current laws in The Netherlands.

\section{References}

Aghajanian GK, Cedarbaum, JM, Wang, RY (1977) Evidence for norepinephrine-mediated collateral inhibition of locus coeruleus neurons. Brain Res 136:570-577

Arnsten AF, Cai JX (1993) Postsynaptic alpha-2 receptor stimulation improves memory in aged monkeys: indirect effects of yohimbine versus direct effects of clonidine. Neurobiol Aging 14:597-603

Aston-Jones G, Rajkowski J, Cohen JD (2000) Locus coeruleus and regulation of behavioral flexibility and attention. Prog Brain Res 126:165-182

Aston-Jones G, Cohen JD (2005) An integrative theory of locus coeruleus-norepinephrine function: adaptive gain and optimal performance. Annu Rev Neurosci 28:403-450

Berridge CW, Waterhouse BD (2003) The locus coeruleus-noradrenergic system: modulation of behavioral state and state-dependent cognitive processes. Brain Res Rev 42:33-84

Bouret S, Sara SJ (2004) Reward expectation, orientation of attention and locus coeruleus-medial frontal cortex interplay during learning. Eur J Neurosci 20:791-802

Chamberlain SR, Muller U, Blackwell AD, Robbins TW, Sahakian BJ (2006) Noradrenergic modulation of working memory and emotional memory in humans. Psychopharmacology 188:397-407

Chun MM, Potter MC (1995) A two-stage model for multiple detection in RSVP. J Exp Psychol Hum Percept Perform 21:109-127

Clark CR, Geffen, GM, Geffen LB (1989) Catecholamines and the covert orientation of attention in humans. Neuropsychologia 27:131-139 
Clayton EC, Rajkowski J, Cohen JD, Aston-Jones G (2004) Phasic activation of monkey locus ceruleus neurons by simple decisions in a forced-choice task. J Neurosci 24:9914-9920

Cohen JD, Aston-Jones G (2005) Cognitive neuroscience: decision amid uncertainty. Nature 436:471-472

Coull JT (1994) Monoamergic modulation of human attentional and executive function. Ph.D. thesis, University of Cambridge, UK

Coull JT, Nobre AC (1998) Where and when to pay attention: the neural systems for directing attention to spatial locations and to time intervals as revealed by both PET and fMRI. J Neurosci 18 : $7426-7435$

Coull JT, Middleton HC, Robbins TW, Sahakian BJ (1995a) Clonidine and diazepam have differential effects on tests of attention and learning. Psychopharmacology 120:322-332

Coull JT, Sahakian BJ, Middleton HC, Young AH, Park SB, McShane RH, Cowen PJ, Robbins TW (1995b) Differential effects of clonidine, haloperidol, diazepam and tryptophan depletion on focused attention and attentional search. Psychopharmacology 121:222-230

Coull JT, Nobre AC, Frith CD (2001) The noradrenergic alpha2 agonist clonidine modulates behavioural and neuroanatomical correlates of human attentional orienting and alerting. Cereb Cortex 11:73-84

Dayan P, Yu AJ (2006) Norepinephrine and neural interrupts. Advances in neural information processing systems 18:243250. MIT, Cambridge, MA

De Martino B, Strange BA, Dolan RJ (2005) Noradrenergic modulation of human attention. Program no. 876.21. 2005 abstract viewer/itinerary planner. Society for Neuroscience, Washington, DC

Fernandez-Duque D, Posner MI (1997) Relating the mechanisms of orienting and alerting. Neuropsychologia 35477-35486

Halliday R, Callaway E, Lannon R (1989) The effects of clonidine and yohimbine on human information processing. Psychopharmacology 99:563-566

Halliday R, Naylor H, Brandeis D, Callaway E, Yano L, Herzig K (1994) The effect of D-amphetamine, clonidine, and yohimbine on human information processing. Psychophysiology 31:331-337

Hommel B, Akyürek EG (2005) Lag-1 sparing in the attentional blink: benefits and costs of integrating two events into a single episode. Q J Exp Psychol A 58:1415-1433

Jouvet M (1969) Biogenic amines and the states of sleep. Science $163: 32-41$
Luteijn F (1966) A new abbreviated Groninger intelligencetest (in Dutch). Nederlands Tijdschrift voor de Psychologie en Haar Grensgebieden 21:675-682

Martens S, Munneke J, Smid H, Johnson A (2006) Quick minds don't blink: electrophysiological correlates of individual differences in attentional selection. J Cogn Neurosci 18:1423-1438

Nieuwenhuis S, Aston-Jones G, Cohen JD (2005a) Decision making, the P3, and the locus coeruleus-norepinephrine system. Psychol Bull 131:510-532

Nieuwenhuis S, Gilzenrat MS, Holmes BD, Cohen JD (2005b) The role of the locus coeruleus in mediating the attentional blink: a neurocomputational theory. J Exp Psychol Gen 134:291-307

Olivers CN, Nieuwenhuis S (2005) The beneficial effect of concurrent task-irrelevant mental activity on temporal attention. Psychol Sci $16: 265-269$

Olivers CNL, Nieuwenhuis S (2006) The beneficial effects of additional task load, positive affect, and instruction on the attentional blink. J Exp Psychol Hum Percept Perform 32:364-379

Olivers CN (2007) The time course of attention: it is better than we thought. Curr Dir Psychol Sci (in press)

Posner MI, Petersen SE (1990) The attention system of the human brain. Annu Rev Neurosci 13:25-42

Posner MI, Snyder CR, Davidson BJ (1980) Attention and the detection of signals. J Exp Psychol 109:160-174

Raymond JE, Shapiro KL, Arnell KM (1992) Temporary suppression of visual processing in an RSVP task: an attentional blink? J Exp Psychol Hum Percept Perform 18:849-860

Robbins TW (1997) Arousal systems and attentional processes. Biol Psychol 45:57-71

Svensson TH, Bunney BS, Aghajanian GK (1975) Inhibition of both noradrenergic and serotonergic neurons in brain by the alphaadrenergic agonist clonidine. Brain Res 92:291-306

Tales A, Butler SR, Fossey J, Gilchrist ID, Jones RW, Troscianko T (2002) Visual search in Alzheimer's disease: a deficiency in processing conjunctions of features. Neuropsychologia 40:1849-1857

Tiplady B, Bowness E, Stien L, Drummond G (2005) Selective effects of clonidine and temazepam on attention and memory. $\mathrm{J}$ Psychopharmacol 19:259-265

Witte EA, Marrocco RT (1997) Alteration of brain noradrenergic activity in rhesus monkeys affects the alerting component of covert orienting. Psychopharmacology 132:315-323

Yu AJ, Dayan P (2005) Uncertainty, neuromodulation, and attention. Neuron 46:681-692 